\title{
Capsaicin enhances anti-proliferation efficacy of pirarubicin via activating TRPV1 and inhibiting PCNA nuclear translocation in 5637 cells
}

\author{
LONG ZHENG, JIAQI CHEN, ZHENKUN MA, WEI LIU, FEI YANG, ZHAO YANG, \\ KE WANG, XINYANG WANG, DALIN HE, LEI LI and JIN ZENG \\ Department of Urology, The First Affiliated Hospital of Xi'an Jiaotong University, Xi'an, Shanxi 710061, P.R. China
}

Received January 26, 2015; Accepted November 6, 2015

DOI: $10.3892 / \mathrm{mmr} .2015 .4623$

\begin{abstract}
The recurrence of bladder cancer after surgery with or without chemotherapy remains a major challenge in bladder cancer treatment. Previous studies have shown that transient receptor potential vanilloid 1 (TRPV1) acts as a tumor suppressor through inducing apoptosis in bladder cancer cells. However, whether activation of TRPV1 has any synergistic effects with pirarubicin (THP), one of main drugs used in urinary bladder instillation chemotherapy to improve chemotherapeutic efficacy has remained elusive. The present study verified that TRPV1 was differentially expressed in bladder cancer cell lines. Furthermore, activation of TRPV1 by capsaicin was shown to induce growth inhibition of 5637 cells in which TRPV1 was highly expressed, while the growth of T24 cells, which express TRPV1 at low levels, was not affected. In addition, the present study demonstrated that activation of TRPV1 enhanced the anti-proliferative effects of pirarubicin using an MTT assay and cell cycle analysis. Finally, immunofluorescent microscopy revealed that activation of TRPV1 prevented the translocation of proliferating cell nuclear antigen to the nucleus. This phenomenon was reversed by pre-treatment with capsazepine, a specific TRPV1 antagonist. In conclusion, the present study confirmed the anti-tumor activity of TRPV1 against bladder cancer. Activation of TRPV1 may be applied as a novel strategy to treat bladder cancer or enhance the therapeutic efficacy of traditional chemotherapeutic drugs.
\end{abstract}

Correspondence to: Professor Jin Zeng or Professor Lei Li, Department of Urology, The First Affiliated Hospital of Xi'an Jiaotong University, 277 Yanta West Road, Xi'an, Shanxi 710061, P.R. China

E-mail: zengjin1984@gmail.com

E-mail: lilydr@163.com

Abbreviations: TRPV1, transient receptor potential vanilloid 1; THP, pirarubicin; CAP, capsaicin; CPZ, capsazepine; PCNA, proliferating cell nuclear antigen

Key words: transient receptor potential vanilloid 1, capsaicin, pirarubicin, chemotherapy, proliferating cell nuclear antigen

\section{Introduction}

Bladder cancer is the most common type of cancer of the urinary system worldwide, with 74,690 novel cases diagnosed and 15,580 mortalities in 2014 (1). It is three times more common in men than in women. Of all bladder cancers, $>80 \%$ are of the superficial, non-invasive type. Intravesical chemotherapy is the first-line treatment for high-risk non-muscle invasive cancer after transurethral resection of the bladder tumor (TURBT) (2). However, $>50 \%$ of patients with superficial bladder cancer undergoing TURBT present with recurrence or develop muscle invasion $(3,4)$. Pirarubicin (THP) is an anthracycline drug, which is widely used for intravesical chemotherapy to treat superficial bladder cancer (5). Pirarubicin has a high affinity for tumor tissue and induces apoptosis of tumor cells by decreasing of the B-cell lymphoma 2 ( $\mathrm{Bcl}-2) / \mathrm{Bcl}-2$-associated $\mathrm{X}$ protein ratio, thereby preventing recurrence of non-muscle invasive bladder cancer. In spite of treatment of bladder cancer patients by TURBT combined with intravesical pirarubicin chemotherapy, the five-year reoccurrence rare remains $>30 \%$ (6). Furthermore, the clinical use of piratubicin is restricted due to potential side effects and cell toxicity (7). Strategies aiming to reduce pirarubicin toxicity and improve the efficacy of pirarubicin chemotherapy are therefore required.

The transient receptor potential vanilloid 1 (TRPV1) channel is a non-selective cation channel, which has an important role in sensory transduction $(8,9)$. TRPV1 has been reported to be expressed not only in bladder nerve fibers but also in non-neuronal tissues $(10,11)$. TRPV1 acts as a tumor suppressor through inducing tumor-cell death and preventing cancer progression upon activation by its agonist (12-14). In human bladder cancer, TRPV1 functions as a tumor suppressor (15). Activation of TRPV1 by capsaicin (Fig. 1A) induces apoptosis and cell cycle arrest, and prevents metastasis of bladder cancer $(16,17)$. Therefore, TRPV1 may be regarded as a therapeutic target for the treatment of bladder cancer. However, whether activation of TRPV1 is able to enhance the therapeutic efficacy of pirarubicin as well as the underlying mechanisms have remained elusive.

The present study assessed the expression of TRPV1 in the 5637 and T24 human bladder cancer cell lines. TRPV1 was 
activated by its agonist, capsaicin, and the effect of TRPV1 activation in pirarubicin-treated cells was investigated using a 3-(4,5-dimethylthiazol-2-yl)-2,5-diphenyltetrazolium bromide (MTT) assay. Cell cycle progression of bladder cancer cells treated with capsaicin and pirarubicin was analyzed by flow cytometry, and the underlying mechanism was explored by immunofluorescence microscopy to prove that TRPV1 activation enhanced the chemotherapeutic effects of pirarubicin.

\section{Materials and methods}

Reagents and antibodies. Capsaicin (molecular weight, $305.42 \mathrm{~g} / \mathrm{mol}$ ), dimethylsulfoxide (DMSO), capsazepine, propidium iodide, RNase A and 4,6-diamidino-2-phenylindole (DAPI) were purchased from Sigma-Aldrich (St. Louis, MO, USA). Capsaicin was dissolved in DMSO to produce a $200 \mathrm{mmol} / \mathrm{l}$ stock solution, which was then further diluted with cell culture medium to the required concentrations. Pirarubicin was kindly donated by Professor Jinhai Fan (Department of Urology, First Hospital of Xi'an Jiaotong University, Xi'an, China). Anti-TRPV1 rabbit polyclonal antibody (cat. no. ab63083; 1:500 dilution) was purchased from Abcam (Cambridge, UK). Anti-proliferating cell nuclear antigen (PCNA) rabbit polyclonal antibody (cat. no. 10205-2-AP; 1;500 dilution) was purchased from Proteintech (Chicago, IL, USA). Anti-glyceraldehyde-phosphate dehydrogenase (GAPDH) antibody was purchased from Santa Cruz Biotechnology, Inc. (Dallas, TX, USA). RPMI-1640 medium and Dulbecco's modified Eagle's medium were purchased from Thermo Fisher Scientific (Waltham, MA, USA). Tris-base, $\mathrm{NaCl}$, sodium dodecyl sulfate (SDS), nonided-P40, sodium deoxycholate, acrylamide-bisacrylamide, ammonium persulfate, tetramethylethylenediamine, Triton X-100, glycerol, bovine serum albumin (BSA) and paraformaldehyde were purchased from Amresco (Solon, OH, USA). Nitrocellulose membranes were purchased from Pall Corporation (Pansacola, FL, USA) and neutral absorbent paper was purchased from Bio-Rad Laboratories, Inc. (Hercules, CA, USA).

Cell culture. The 5637 and T24 human bladder transitional cell carcinoma cell lines were obtained from the American Type Culture Collection (Manassas, VA, USA). These two cell lines were cultured at $37^{\circ} \mathrm{C}$ and in a humidified atmosphere containing $5 \% \mathrm{CO}_{2}$. The 5637 cells were cultured in RPMI-1640 with $10 \%$ fetal bovine serum (FBS) (Gibco; Thermo Fisher Scientific) supplemented with $1 \%$ penicillin-streptomycin, while T24 cells were cultured in Dulbecco's modified Eagle's medium with $10 \%$ FBS and $1 \%$ penicillin-streptomycin.

Cell proliferation assay. Cell viability was analyzed using an MTT assay. A total of $8 \times 10^{3}$ cells per well were seeded in 96-well culture plates and incubated for $12 \mathrm{~h}$ for attachment. The cells were then treated with TRPV1 agonist capsaicin $(0$, $50,100,150$ and $200 \mu \mathrm{M}$ ) alone for $12 \mathrm{~h}$ alone or capsaicin $(0,50,100,150$ and $200 \mu \mathrm{M})$ following pre-incubation with TRPV1 antagonist capsazepine $(10 \mu \mathrm{M})$ for $2 \mathrm{~h}$. Following washing, the cells were incubated with $20 \mu \mathrm{l}$ MTT solution $\left(0.5 \mathrm{mg} / \mathrm{ml}\right.$ in culture medium) at $37^{\circ} \mathrm{C}$ for $4 \mathrm{~h}$. The medium was carefully discarded and DMSO $(150 \mu \mathrm{l})$ was added to dissolve the formazan crystals. The absorbance of the wells was then measured at a wavelength of $490 \mathrm{~nm}$ using the Microplate Autoreader (Bio-Tek Instruments Inc., Winooski, VT, USA). The assay was performed as three independent experiments.

Flow cytometric analysis. A total of $2 \times 10^{5}$ cells per well were seeded in 6-well culture plates and incubated for $12 \mathrm{~h}$ for attachment. For cell cycle analysis, 5637 cells were treated for $12 \mathrm{~h}$ as described above. Following harvesting and washing with cold phosphate-buffered saline (PBS), cells were fixed overnight at $-20^{\circ} \mathrm{C}$ in $70 \%$ ethanol. Prior to analysis, cells were washed with PBS and incubated with $50 \mu \mathrm{g} / \mathrm{ml}$ propidium iodide and $50 \mu \mathrm{g} / \mathrm{ml}$ RNase A in PBS at room temperature for $30 \mathrm{~min}$. Flow cytometric analysis was then performed using a FACSCalibur (BD Biosciences, Franklin Lakes, NJ, USA) with CellQuest software version 3.3 (BD Biosciences). ModFit LT software (Version 3.0; Verity Software House, Topsham, ME, USA) was used to calculate the cell cycle distributions.

Immunofluorescence. Following treatment of 5637 cells attached to cover slips as specified above for $12 \mathrm{~h}$, cells were rinsed with PBS and fixed with $4 \%$ paraformaldehyde for $15 \mathrm{~min}$ at room temperature. Cells were then washed three times with PBS and incubated with $0.1 \%$ Triton X-100 for $15 \mathrm{~min}$ to increase permeability of the cell membrane. Following a further three washes with PBS, cells were incubated for 60 min with 5\% BSA in PBS to block unspecific binding of antibodies. Anti-PCNA antibodies diluted in 1\% BSA (1:50) were applied and cells were incubated overnight at $4^{\circ} \mathrm{C}$. Following three washes with PBS, cells were incubated with the secondary antibody diluted in $1 \%$ BSA for $60 \mathrm{~min}$ at room temperature. Cell nuclei were counterstained using $1 \mu \mathrm{g} / \mathrm{ml}$ DAPI for $3 \mathrm{~min}$. Following three more washes, coverslips were mounted on microscopy slides in $50 \%$ glycerol. Fluorescence microscopy (U-LH100-3; Olympus, Tokyo, Japan) was used to observe the PCNA distribution patterns.

Reverse-transcription quantitative polymerase chain reaction (RT-qPCR). Total RNA of 5637 and T24 cells was extracted using the RNAfast200 kit (Feijie Biotechnology, Shanghai, China) and quantitated by measuring its absorbance at $260 \mathrm{~nm}$ using a Microplate Autoreader (Bio-Tek Instruments, Inc.). RNA (500 ng) was reverse-transcribed using PrimerScript ${ }^{\mathrm{TM}}$ RT Master Mix (Perfect Real Time; Takara Biotechnology Co., Ltd., Dalian, China). 5X PrimeScript RT Master mix $(2 \mu \mathrm{l})$, RNase-free $\mathrm{dH}_{2} \mathrm{O}(7 \mu \mathrm{l})$ and total RNA $(1 \mu \mathrm{l})$ were mixed and reacted at $37^{\circ} \mathrm{C}$ for $16 \mathrm{~min}$ and $85^{\circ} \mathrm{C}$ for $5 \mathrm{sec}$. Real-time PCR was then performed using the SYBR Premix Ex Taq ${ }^{\mathrm{TM}}$ II system (Takara Biotechnology Co., Ltd., Dalian, China) and the Bio-Rad CFX96 ${ }^{\mathrm{TM}}$ Real-time system (Bio-Rad Laboratories, Inc.). SYBR Premix Ex Taq II (12.5 $\mu 1), 1 \mu$ l sense primer $(10 \mu \mathrm{M}), 1 \mu \mathrm{l}$ anti-sense primer $(10 \mu \mathrm{M}) 2 \mu \mathrm{l}$ cDNA solution and $8.5 \mu \mathrm{l}$ RNase-free water were mixed together. The following thermocycling protocol was used with three stages, including pre-degeneration for $95^{\circ} \mathrm{C}$ for $30 \mathrm{sec}$, one repeat; PCR amplification at $95^{\circ} \mathrm{C}$ for $5 \mathrm{sec}$ followed by $60^{\circ} \mathrm{C}$ for $30 \mathrm{sec}, 40$ repeats; and dissociation at $95^{\circ} \mathrm{C}$ for $15 \mathrm{sec}$ followed by $60^{\circ} \mathrm{C}$ for $30 \mathrm{sec}$ and $95^{\circ} \mathrm{C}$ for $15 \mathrm{sec}$. GAPDH was used as the internal control. The following primers were used: TRPV1 sense, 5'-GCTCAG CCCGAGGAAGTTT-3' and anti-sense, 5'-ACTCTTGAAGAC 

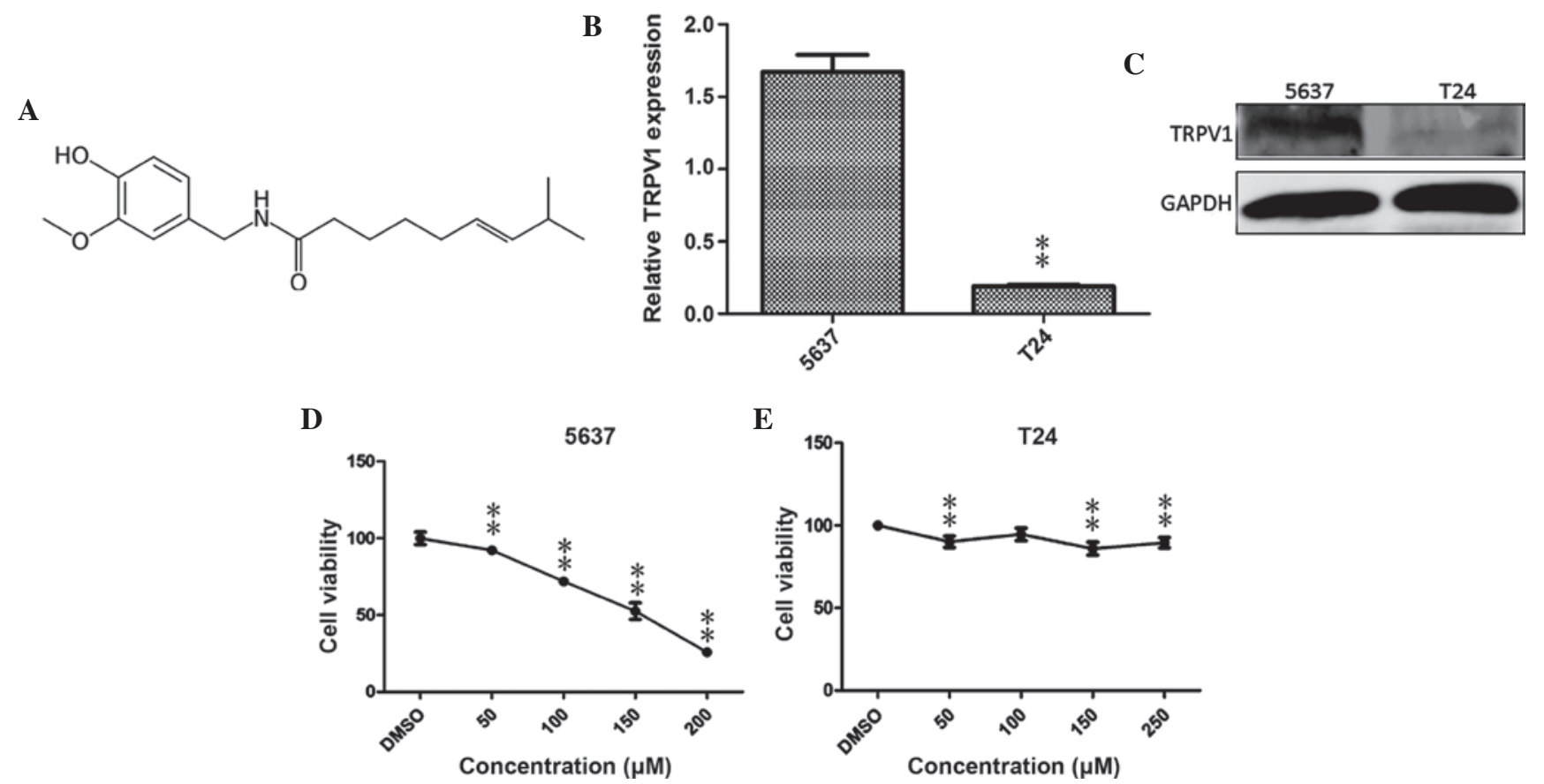

Figure 1. Capsaicin induces growth inhibition in bladder cancer cells with high expression of TRPV1. (A) Chemical structure of TRPV1 agonist capsaicin. (B) Relative TRPV1 mRNA levels in 5637 and T24 cells. ${ }^{* *} \mathrm{P}<0.01$ vs. 5637. (C) Western blot analysis of TRPV1 in cell lysates from 5637 and T24 cells. (D and E) An MTT assay was used to assess the effect of capsaicin on the viability of 5637 and T24 bladder cancer cells. Values are expressed as the mean \pm standard error from three independent experiments. ${ }^{* *} \mathrm{P}<0.01$ vs. DMSO group. DMSO, dimethyl sulfoxide; TRPV1, transient receptor potential vanilloid 1.

CTCAGCGTCC-3' (18); PCNA sense, 5'-CCTGCTGGG ATA TTAGCTCCA-3' and anti-sense, 5'-CAGCGGTAGGTGTCG AAGC-3'; GAPDH sense, 5'-ATGGGGAAGGTGAAGGTC GG-3' and anti-sense, 5'-GACGGTGCCATG GAATTTGC-3'.

Western blot analysis. Following capsaicin treatment as stated above, cells were harvested and washed with cold PBS three times. Total cellular protein lysates were prepared with radioimmunoprecipitation assay buffer [50 $\mathrm{mM}$ Tris (pH 8.0), $150 \mathrm{mM} \mathrm{NaCl}, 0.1 \%$ SDS, $1 \%$ nonided-P40 and $0.5 \%$ sodium deoxycholate] including proteinase inhibitors (1\% cocktail and $1 \mathrm{mM}$ phenylmethanesulfonyl fluoride, both from Sigma-Aldrich). The lysates were then centrifuged at $20,000 \mathrm{x}$ g for $15 \mathrm{~min}$ at $4^{\circ} \mathrm{C}$ and the supernatants were collected for determination of the protein concentration. The content of protein was quantified using an Enhanced BCA Protein Assay kit (Beyotime Institute of Biotechnology, Haimen, China). For western blot analysis, $30 \mu \mathrm{g}$ protein per lane was separated by 10 or $12 \%$ SDS-polyacrylamide gel electrophoresis and transferred onto nitrocellulose membranes. The membranes were blocked with $5 \%$ skimmed milk in Tris-buffered saline ( $\mathrm{pH} 7.6$; TBS) at room temperature for $1 \mathrm{~h}$. Membranes were then incubated with primary antibodies in 5\% skimmed milk in TBS at $4^{\circ} \mathrm{C}$ overnight, followed by washing with TBS containing Tween-20. Goat anti-rabbit (cat. no. 926-32211) or goat anti-mouse secondary antibodies (cat. no. 926-68070) (LI-COR Biosciences, Lincoln, NE, USA) diluted at 1:1000 in 5\% skimmed milk were then applied, followed by incubation at room temperature in the dark for $1 \mathrm{~h}$. Subsequent to washing as described above in the dark, membranes were dried with neutral absorbent paper and scanned using the Odyssey detection system (LI-COR Biosciences). GAPDH was used as the loading control.
Statistical analysis. Values are expressed as the mean \pm standard error from three independent experiments. Statistical analysis was performed using Student's t-test with SPSS 13.0 (SPSS Inc., Chicago, IL, USA). P<0.05 was considered to indicate a statistically significant difference.

\section{Results}

Capsaicin inhibits the growth of bladder cancer cells with high TRPVI expression. The results of previous studies indicated that TRPV1 is abundantly expressed at low levels in a variety of non-neural tissues (19-21). The present study first verified the expression of TRPV1 in superficial bladder cancer cells. The 5637 and T24 cell lines were selected to be analyzed for TRPV1 expression at the mRNA and protein level using RT-qPCR and western blot analysis, respectively. In 5637 cells, TRPV1 was highly expressed at the mRNA and protein level, while its expression was low in T24 cells (Fig. 1B and C). These results indicated that TRPV1 was differentially expressed in human superficial bladder cancer cell lines. In order to determine the function of TRPV1 in the two cell lines, capsaicin, a specific agonist of TRPV1, was applied at various concentrations $(0,50,100,150$ and $200 \mu \mathrm{M})$ for $48 \mathrm{~h}$. The viability of 5637 and T2 4 cells was then detected using an MTT assay. The results showed that 5637 cells were sensitive to capsaicin treatment $\left(\mathrm{IC}_{50}, 149 \mu \mathrm{M}\right)$ (Fig. 1D), while $\mathrm{T} 24$ cells were insensitive $\left(\mathrm{IC}_{50}, 266 \mu \mathrm{M}\right)$ (Fig. 1E). Capsaicin exerted significant growth inhibitory effects on 5637 cells that highly express TRPV1; however, this phenomenon was not observed in T24 cells, which express TRPV1 at low levels.

Capsaicin enhances the anti-proliferative effects of pirarubicin in 5637 cells. Next, the present study explored the 
A<smiles>COc1cccc2c1C(=O)c1c(O)c3c(c(O)c1C2=O)C[C@@](O)(C(=O)CO)C[C@@H]3OC1CC2CC(OC3CCCCO3)C(N)C(C2)O1</smiles>

B

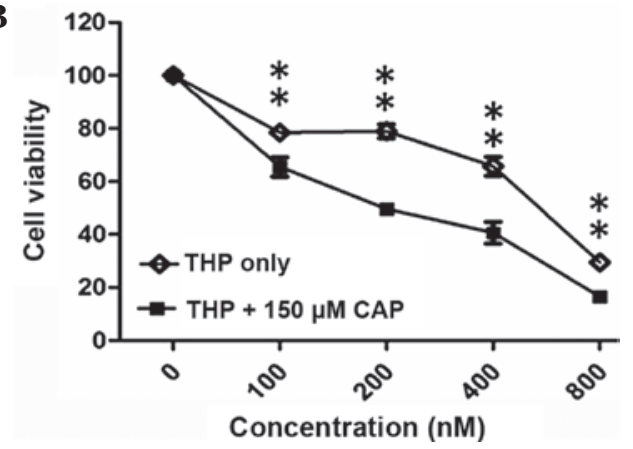

Figure 2. Capsaicin enhances the anti-proliferative effects of pirarubicin in 5637 cells. (A) Chemical structure of pirarubicin. (B) An MTT assay was used to assess the viability of 5637 cells treated by pirarubicin alone or combination of capsaicin and pirarubicin. Values are expressed as the mean \pm standard error from three independent experiments. ${ }^{* *} \mathrm{P}<0.01$. THP, pirarubicin; CAP, capsaicin.

A

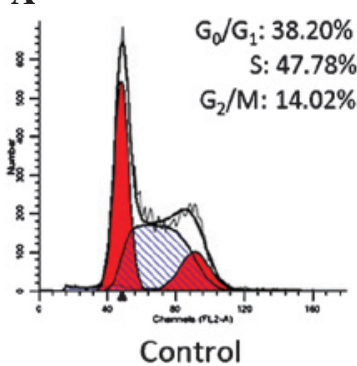

C

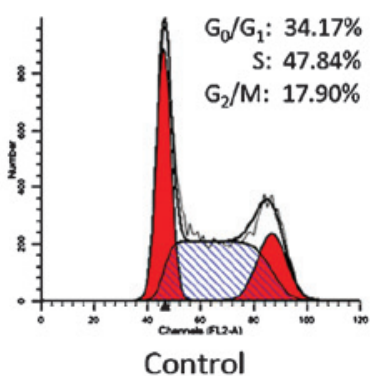

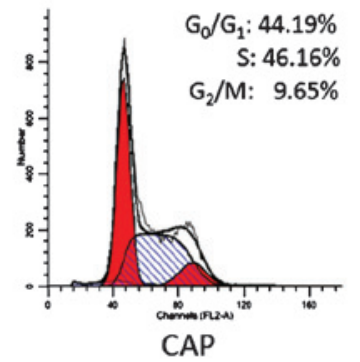

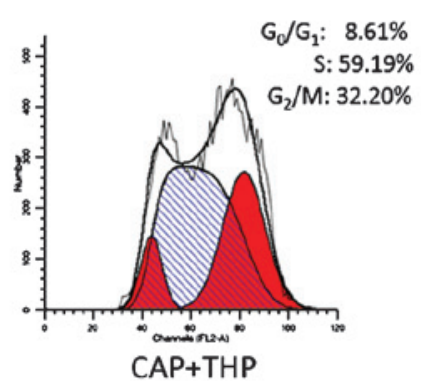

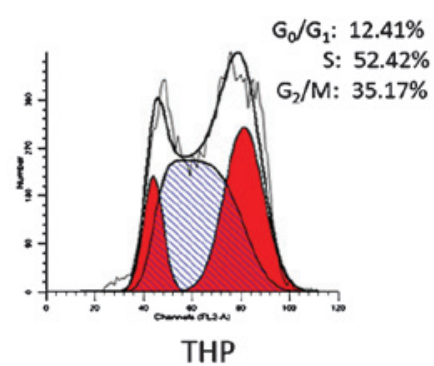

THP

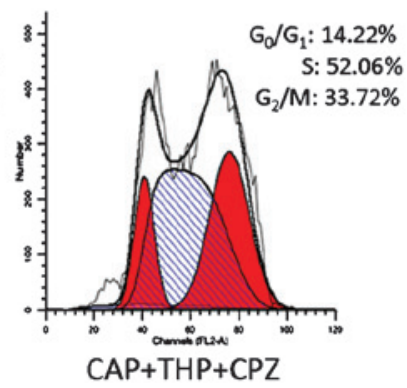

B

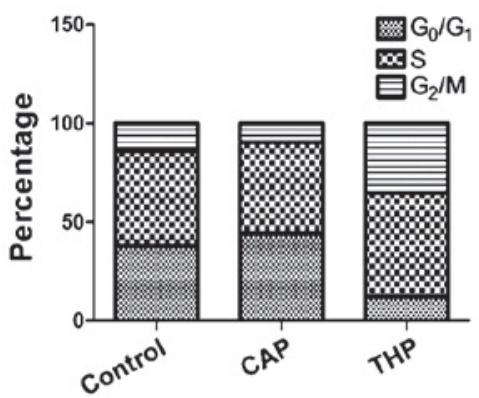

D

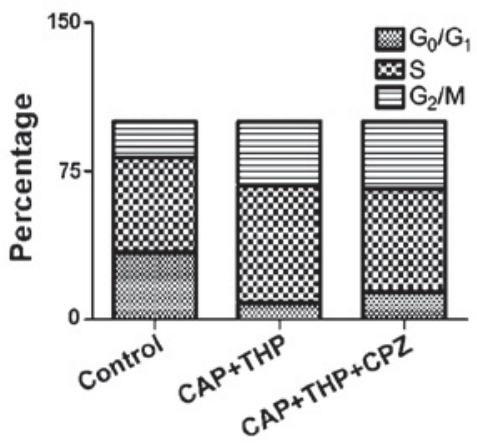

Figure 3. Combination of pirarubicin and TRPV1 agonist capsaicin induces S-phase arrest in 5637 cells. (A) 5637 cells in logarithmic growth phase were treated with $400 \mathrm{nM}$ pirarubicin alone or $150 \mu \mathrm{M}$ capsaicin alone for $12 \mathrm{~h}$ and the cell cycle was analyzed by flow cytometry. (B) Quantification of cell-cycle populations in A. (C) 5637 cells were treated with $400 \mathrm{nM}$ pirarubicin combined with $150 \mu \mathrm{M}$ capsaicin with or without $20 \mu \mathrm{M}$ capsazepine. (D) Quantification of cell-cycle populations in C. THP, pirarubicin; CAP, capsaicin; CPZ, capsazepine.

implication of TRPV1 in the therapeutic effects of pirarubicin. Following incubation of 5637 cells with various concentrations of pirarubicin with or without TRPV1 agonist capsaicin $(150 \mu \mathrm{M})$, a cell viability assay was performed. The $\mathrm{IC}_{50}$ was $566 \mathrm{nM}$ in the group treated with pirarubicin alone, while the $\mathrm{IC}_{50}$ was $335 \mathrm{nM}$ in the group treated with pirarubicin and capsaicin (Fig. 2B). These results demonstrated that the anti-proliferative effects of pirarubicin were enhanced by capsaicin-mediated activation of TRPV1. Furthermore, the results indicated that $200 \mathrm{nM}$ pirarubicin combined with $150 \mu \mathrm{M}$ capsaicin produced a greater anti-proliferative effect than $400 \mathrm{nM}$ pirarubicin treatment alone. Therefore, activation of TRPV1 enhanced the growth-inhibitory effects of pirarubicin and sensitized bladder cancer cells to pirarubicin.

Combination of pirarubicin and TRPVI agonist capsaicin induces S-phase and G2/M-phase arrest in 5637 cells. As a member of the anthracycline family, pirarubicin intercalates between base pairs of DNA strands, thereby preventing the replication of DNA and causing cell-cycle arrest in S- or G2/M-phase (22-24). To further explore the mechanisms by which TRPV1 sensitizes bladder cancer cells to pirarubicin treatment, the effects of pirarubicin and capsaicin on their cell cycle distribution was assessed using flow cytometry. The results showed that, compared with the control group, capsaicin $(150 \mu \mathrm{M})$ elevated the percentage of cells in $\mathrm{G} 0 / \mathrm{G} 1$ phase and decreased the percentage of cells G2/M phase. By contrast, pirarubicin increased the S- and G2/M-phase populations and reduced the G0/G1-phase population (Fig. 3A and B). Furthermore, the combined effects of the two drugs on the cell cycle progression were assessed. When TRPV1 was activated by capsaicin and pirarubicin was simultaneously applied for $12 \mathrm{~h}$, the S- and G2/M-phase populations were significantly elevated, while the G0/G1 population was decreased, 
A

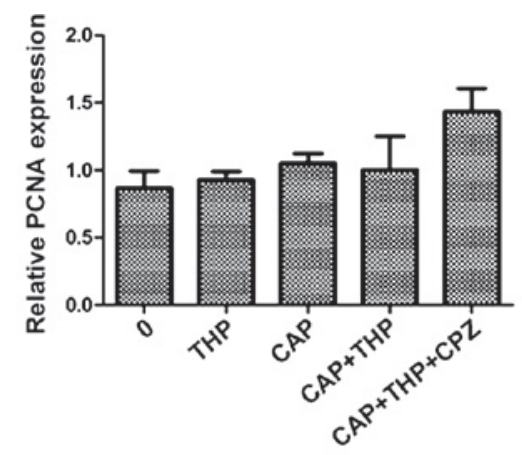

C
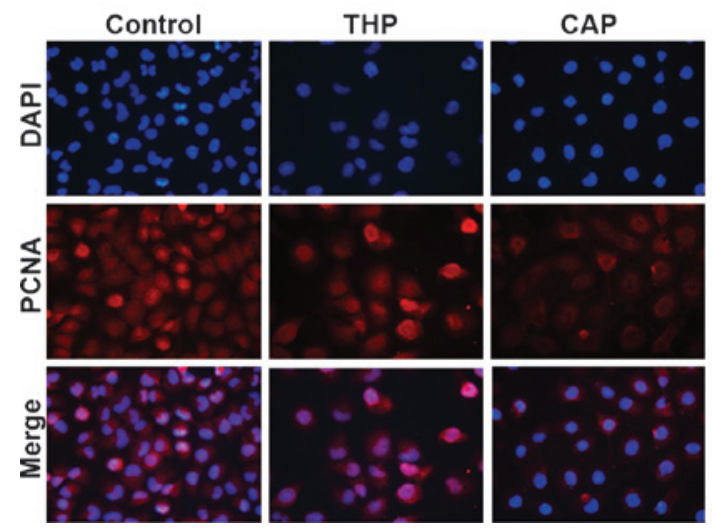

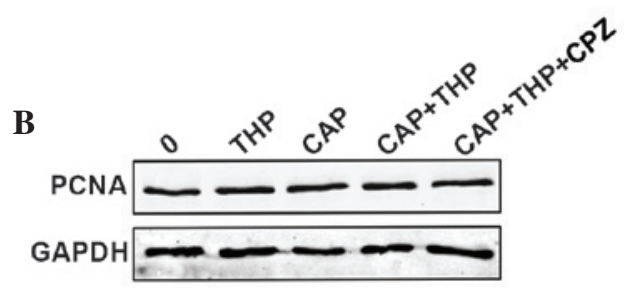

D
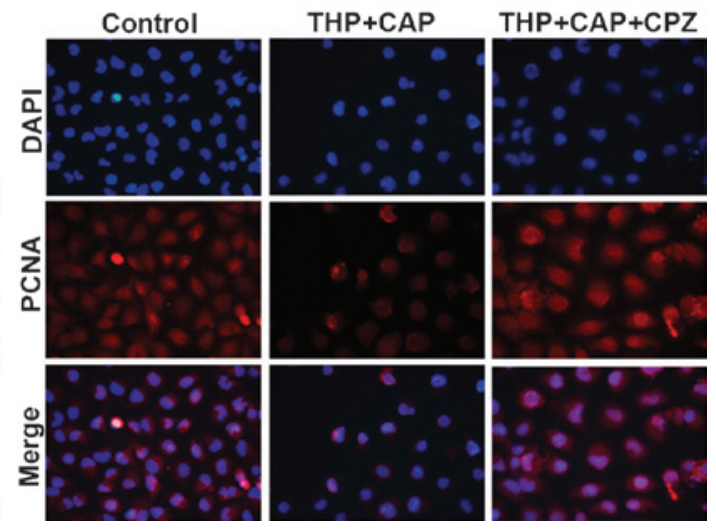

Figure 4. Combined treatment with pirarubicin and TRPV1 agonist capsaicin inhibits PCNA translocation to the nucleus in 5637 cells. (A) Relative mRNA expression of PCNA in whole-cell lysates of each treatment group was assessed by reverse-transcription quantitative polymerase chain reaction analysis. Values are expressed as the mean \pm standard error from three independent experiments. No significant differences were present between any of the groups. (B) Expression of PCNA at the protein level in whole-cell lysates of each treatment group was assessed by western blotting. A blot representative of three experiments is shown; no marked effects on PCNA expression was observed. (C and D) Distribution patterns of PCNA in each indicated treatment group were visualized by immunofluorescence (magnification, x400). THP, pirarubicin; CAP, capsaicin; CPZ, capsazepine; TRPV1, transient receptor potential vanilloid 1 ; PCNA, proliferating cell nuclear antigen.

compared with those in the control group; however, treatment with the TRPV1 antagonist capsazepine partly reversed this $\mathrm{S}$-phase arrest induced by combined treatment with capsaicin and pirarubicin (Fig. 3C and D).

Combined treatment with pirarubicin and TRPVI agonist capsaicin inhibits PCNA translocation to the nucleus. PCNA is a well-conserved $36-\mathrm{kDa}$ nuclear protein that exists in almost all types of eukaryotic cell (25). PCNA is an important auxiliary protein of DNA polymerase $\delta$ and is required for DNA synthesis and DNA repair in the $S$ phase of the cell cycle $(26,27)$. In order to investigate whether the observed S- and G2/M-phase arrest induced by combined treatment with pirarubicin and TRPV1 agonist capsaicin was caused by any interaction with PCNA, RT-qPCR and western blot analysis were performed to detect PCNA. The results showed that the expression of PCNA was slightly, but not significantly, elevated by treatment with pirarubicin, capsaicin and their combination. of note, further addition of capsazepine elevated the PCNA levels; however, effect was proved not to be significant (Fig. 4A and B). Furthermore, the effects of the drugs on nuclear translocation of PCNA were assessed by immunofluorescence microscopy. In the control group, PCNA was expressed in the nucleus and cytoplasm, which was not slightly increased by pirarubicin treatment (Fig. 4C). However, treatment with TRPV1 agonist capsaicin led to reduced levels of PCNA in the nucleus, and a similar phenomenon was observed in cells treated with a combination of pirarubicin and capsaicin. However, application of TRPV1 antagonist capsazepine reversed the inhibitory effects of the combination of capsaicin and pirarubicin on the nuclear translocation of PCNA (Fig. 4D).

\section{Discussion}

Although chemotherapy of superficial bladder cancer is efficient, the high rate of recurrence remains the most significant challenge $(3,4)$. The present study reported TRPV1 as a novel target for the treatment bladder cancer or the sensitization of bladder cancer cells to chemotherapy. TRPV1 agonist capsaicin was shown to inhibit the growth of bladder cancer cells that highly expressed TRPV1. Previous studies have proved that TRPV1 is expressed in bladder cells and functions as a tumor suppressor in bladder carcinoma (15). Kalogris et al (28) demonstrated that TRPV1 was expressed in neoplastic bladder tissues and the progression of bladder cancer was associated with a marked decrease of TRPV1 expression. Capsaicin, which is regarded as a tumor suppressor, was shown to induce apoptosis in bladder cancer through activating dendritic cells and halting cell-cycle progression (29-31), while the role of TRPV1 has been studied less extensively. The present study confirmed that TRPV1 was differentially expressed in two bladder cancer cell lines derived from bladder cancer tissues at different stages. Furthermore, capsaicin was demonstrated to inhibit the proliferation of 
5637 cells, in which TRPV1 was highly expressed, while it did not markedly affect the proliferation of T24 cells, which exhibited low expression of TRPV1. These results indicated that the anti-proliferative and tumor-suppressive activities of capsaicin were dependent on TRPV1.

The cell cycle is a highly organized process by which eukaryotic cells replicate $(32,33)$. Blocking of cell-cycle progression directly inhibits cell growth, and the majority of drugs used for intravesical instillation therapy of bladder cancer, including doxorubicin, cisplatin and mitomycin $\mathrm{C}$, target various stages of the cell cycle to inhibit cell-cycle progression. In the present study, pirarubicin, a chemotherapeutic drug of the anthracycline family, was shown to induce cell-cycle arrest in G2/M phase, while TRPV1 agonist capsaicin halted cell-cycle progression in G0/G1 phase. TRPV1 activation is associated with cell-cycle arrest (34). Treatment of RT4 low-grade urothelial cancer cells with the specific TRPV1 agonist capsaicin induced a TRPV1-dependent cell-cycle arrest in G0/G1 phase by modulating p53, p21 and cyclin-dependent kinase 2 (Cdk2) (34). However, Mori et al (35) also reported that capsaicin caused cell-cycle arrest in prostate cancer cells in a TRPV1-independent manner. The present study further proved that combined application of pirarubicin and capsaicin led to significant S-phase arrest, which, however, could be reversed by TRPV1 antagonist capsazepine. This result confirmed that activation of TRPV1 enhanced pirarubicin-induced cell-cycle arrest.

In the $\mathrm{S}$ phase of the cell cycle, PCNA acts in a slide clamp-like manner on the DNA polymerase delta and is also regarded as a component of the S-phase checkpoint (36). Koundrioukoff et al (37) demonstrated that PCNA combines with cyclin A and Cdk2 to form a complex, which was able to phosphorylate the PCNA binding site of the large sub-unit of replication factor $\mathrm{C}$ as well as DNA ligase I. Thus, PCNA nuclear translocation facilitates cell-cycle progression from $\mathrm{S}$ phase to G2/M phase. Furthermore, PCNA is crucial for the establishment of cohesion proteins and controlled sister chromatid cohesion by interacting with Eco1, also named as Ctf7, which is another essential protein during $\mathrm{S}$ phase (38). For these reasons, PCNA is indispensable in cancer-cell growth, proliferation and cell-cycle progression. Furthermore, PCNA may be used as a diagnostic or prognostic factor for bladder cancer $(39,40)$. Yoshikawa et al (39) indicated that detection of PCNA expression by immunohistochemistry may be used for estimating the malignant potential of bladder cancer. High expression of PCNA has been shown to be associated with poor prognosis of patients with bladder cancer (40). In addition, PCNA represents a target for bladder cancer therapy. Tong et al (41) reported that transfection of an anti-sense RNA targeting PCNA produced growth inhibitory effects and G0/G1-phase arrest in bladder cancer. Furthermore, Zhu et al (42) found that PCNA anti-sense oligonucleotides in combination with recombinant adenovirus p53 suppressed the growth of human bladder cancer. Of note, previous studies have indicated that TRPV1 negatively regulates the expression and activity of PCNA. Expression of PCNA in cultured human outer root sheath keratinocytes, which expressed TRPV1, was decreased by nearly $30 \%$ (43). Low expression of PCNA was also observed in intestinal epithelial tissues obtained from TRPV1 knockout mice (44). In the present study, pirarubicin slightly enhanced the nuclear translocation of PCNA; however, activation of TRPV1 by capsaicin blocked the nuclear translocation of PCNA. Combined application of these two drugs led to a marked loss of PCNA nuclear translocation. However, this phenomenon was reversed by treatment with TRPV1 antagonist capsazepine. Thus, the results of the present study proved that activation of TRPV1 by capsaicin prevented PCNA translocation to the nucleus, which was reversed by inhibition of TRPV1. As no significant changes in the mRNA and protein levels of PCNA were observed, it was indicated that capsaicin treatment for $12 \mathrm{~h}$ was insufficient to degrade PCNA protein or mRNA. Further study is currently undertaken in our group to elucidate the mechanisms of the inhibition of PCNA nuclear translocation induced by TRPV1 activation.

In conclusion, the present study revealed that TRPV1 agonist capsaicin induced growth arrest in bladder cancer cells which expressed TRPV1 at high levels. Application of capsaicin increased the chemotherapeutic efficacy of pirarubicin through inducing S-phase arrest. Furthermore, it was demonstrated that activation of TRPV1 by capsaicin blocked nuclear translocation of PCNA, which was reversed by a TRPV1 antagonist. These results indicated that TRPV1 may be used as a target for the treatment of bladder cancer or for enhancing the chemotherapeutic effects of traditional chemotherapeutic drugs.

\section{Acknowledgements}

This study was supported by the National Natural Science Foundation of China (grant nos. 81472679 to LL and 81101936 to JZ).

\section{References}

1. Siegel R, Ma J, Zou Z and Jemal A: Cancer statistics, 2014. CA A Cancer J Clin 64: 9-29, 2014.

2. Cookson MS, Chang SS, Lihou C, Li T, Harper SQ, Lang Z and Tutrone RF: Use of intravesical valrubicin in clinical practice for treatment of nonmuscle-invasive bladder cancer, including carcinoma in situ of the bladder. Ther Adv Urol 6: 181-191,2014.

3. Kamat AM and Lamm DL: Antitumor activity of common antibiotics against superficial bladder cancer. Urology 63: 457-460, 2004.

4. Arakawa M, Nakamura K, Yamada Y, Kato K, Katsuda R, Tobiume M, Zennami K, Watanabe M, Kato Y, Nishikawa G, et al: Intravesical administration of pirarubicin against superficial bladder cancer: Relationship between tumor tissue concentration and exposure time in the bladder or therapeutic effect. Exp Ther Med 2: 901-905, 2011.

5. Fukushima T, Ueda T and Nakamura T: Pharmacokinetics and action mechanism of anthracyclines. Jpn J Cancer Chemother 19: 445-450, 1992 (In Japanese).

6. Miki T, Nonomura N, Kojima Y, Okuyama A, Nakano E, Kiyohara H, Fujioka H, Koide T, Wakatsuki A, Kuroda H, et al: A randomized study on intravesical pirarubicin (THP) chemoprophylaxis of recurrence after transurethral resection of superficial bladder cancer. Acta Urol Jpn 43: 907-912, 1997 (In Japanese).

7. Zhou J, Zhang X, Li M, Wu W, Sun X, Zhang L and Gong T: Novel lipid hybrid albumin nanoparticle greatly lowered toxicity of pirarubicin. Mol Pharm 10: 3832-3841, 2013.

8. Cao E, Cordero-Morales JF, Liu B, Qin F and Julius D: TRPV1 channels are intrinsically heat sensitive and negatively regulated by phosphoinositide lipids. Neuron 77: 667-679, 2013.

9. Ho KW, Ward NJ and Calkins DJ: TRPV1: A stress response protein in the central nervous system. Am J Neurodegener Dis 1: 1-14, 2012.

10. Avelino A and Cruz F: TRPV1 (vanilloid receptor) in the urinary tract: Expression, function and clinical applications. Naunyn Schmiedebergs Arch Pharmacol 373: 287-299, 2006. 
11. Lazzeri M, Vannucchi MG, Spinelli M, Bizzoco E, Beneforti P, Turini D and Faussone-Pellegrini MS: Transient receptor potential vanilloid type 1 (TRPV1) expression changes from normal urothelium to transitional cell carcinoma of human bladder. Eur Urol 48: 691-698, 2005.

12. Vercelli C, Barbero R, Cuniberti B, Racca S, Abbadessa G, Piccione $\mathrm{F}$ and Re G: Transient receptor potential vanilloid 1 expression and functionality in mcf-7 cells: A preliminary investigation. J Breast Cancer 17: 332-338, 2014.

13. Wu TT, Peters AA, Tan PT, Roberts-Thomson SJ and Monteith GR: Consequences of activating the calcium-permeable ion channel TRPV1 in breast cancer cells with regulated TRPV1 expression. Cell Calcium 56: 59-67, 2014.

14. Stock K, Kumar J, Synowitz M, Petrosino S, Imperatore R Smith ES, Wend P, Purfürst B, Nuber UA, Gurok U, et al: Neural precursor cells induce cell death of high-grade astrocytomas through stimulation of TRPV1. Nat Med 18: 1232-1238, 2012.

15. Mistretta F, Buffi NM, Lughezzani G, Lista G, Larcher A, Fossati N, Abrate A, Dell'Oglio P, Montorsi F, Guazzoni G and Lazzeri M: Bladder cancer and urothelial impairment: The role of TRPV1 as potential drug target. Biomed Res Int 2014: 987149 , 2014.

16. Amantini C, Ballarini P, Caprodossi S, Nabissi M, Morelli MB, Lucciarini R, Cardarelli MA, Mammana G and Santoni G: Triggering of transient receptor potential vanilloid type 1 (TRPV1) by capsaicin induces Fas/CD95-mediated apoptosis of urothelial cancer cells in an ATM-dependent manner. Carcinogenesis 30: 1320-1329, 2009.

17. Santoni G, Caprodossi S, Farfariello V, Liberati S, Gismondi A and Amantini C: Antioncogenic effects of transient receptor potential vanilloid 1 in the progression of transitional urothelial cancer of human bladder. ISRN Urol 2012: 458238, 2012.

18. Sanchez MG, Sanchez AM, Collado B, Malagarie-Cazenave S, Olea N, Carmena MJ, Prieto JC and Diaz-Laviada II: Expression of the transient receptor potential vanilloid 1 (TRPV1) in LNCaP and $\mathrm{PC}-3$ prostate cancer cells and in human prostate tissue. Eur J Pharmacol 515: 20-27, 2005.

19. Costa MA, Fonseca BM, Keating E, Teixeira NA and Correia-da-Silva G: Transient receptor potential vanilloid 1 is expressed in human cytotrophoblasts: Induction of cell apoptosis and impairment of syncytialization. Int J Biochem Cell Biol 57: $177-185,2014$

20. Lang H, Li Q, Yu H, Li P, Lu Z, Xiong S, Yang T, Zhao Y, Huang X, Gao P, et al: Activation of TRPV1 attenuates high salt-induced cardiac hypertrophy through improvement of mitochondrial function. Br J Pharmacol Oct 23, 2014 (Epub ahead of print).

21. Coelho A, Wolf-Johnston AS, Shinde S, Cruz CD, Cruz F, Avelino A and Birder LA: Urinary bladder inflammation induces Changes in urothelial nerve growth factor and TRPV1 channels. Br J Pharmacol 172: 1691-1699, 2015.

22. Kizek R, Adam V, Hrabeta J, Eckschlager T, Smutny S, Burda JV, Frei E and Stiborova M: Anthracyclines and ellipticines as DNA-damaging anticancer drugs: Recent advances. Pharmacol Ther 133: 26-39, 2012

23. Zheng SE, Xiong S, Lin F, Qiao GL, Feng T, Shen Z, Min DL, Zhang CL and Yao Y: Pirarubicin inhibits multidrug-resistant osteosarcoma cell proliferation through induction of $\mathrm{G} 2 / \mathrm{M}$ phase cell cycle arrest. Acta Pharmacol Sin 33: 832-838, 2012.

24. Takimoto $\mathrm{CH}$ and Calvo E: Principles of oncologic pharmacotherapy. Cancer Management: A Multidisciplinary Approach 11, 2008.

25. Garcia RL, Coltrera MD and Gown AM: Analysis of proliferative grade using anti-PCNA/cyclin monoclonal antibodies in fixed, embedded tissues. Comparison with flow cytometric analysis. Am J Pathol 134: 733-739, 1989.

26. Bravo R, Frank R, Blundell PA and Macdonald-Bravo $\mathrm{H}$ Cyclin/PCNA is the auxiliary protein of DNA polymerase-delta. Nature 326: 515-517, 1987.

27. Kelman Z: PCNA: Structure, functions and interactions. Oncogene 14: 629-640, 1997.
28. Kalogris C, Caprodossi S, Amantini C, Lambertucci F, Nabissi M, Morelli MB, Farfariello V, Filosa A, Emiliozzi MC, Mammana G and Santoni G: Expression of transient receptor potential vanilloid-1 (TRPV1) in urothelial cancers of human bladder: Relation to clinicopathological and molecular parameters. Histopathology 57: 744-752, 2010.

29. Chen D, Yang Z, Wang Y, Zhu G and Wang X: Capsaicin induces cycle arrest by inhibiting cyclin-dependent-kinase in bladder carcinoma cells. Int J Urol 19: 662-668, 2012.

30. Gilardini Montani MS, D'Eliseo D, Cirone M, Di Renzo L, Faggioni A, Santoni A and Velotti F: Capsaicin-mediated apoptosis of human bladder cancer cells activates dendritic cells via CD91. Nutrition 31: 578-581, 2015.

31. D'Eliseo D, Manzi L and Velotti F: Capsaicin as an inducer of damage-associated molecular patterns (DAMPs) of immunogenic cell death (ICD) in human bladder cancer cells. Cell Stress Chaperones 18: 801-808, 2013.

32. Hartwell LH and Kastan MB: Cell cycle control and cancer. Science 266: 1821-1828, 1994.

33. Graña X and Reddy EP: Cell cycle control in mammalian cells Role of cyclins, cyclin dependent kinases (CDKs), growth suppressor genes and cyclin-dependent kinase inhibitors (CKIs). Oncogene 11: 211-219, 1995.

34. Li Q, Wang XH, Yang ZH, Wang HP, Yang ZW, Li SW and Zheng XM: Induction of cell cycle arrest in bladder cancer RT4 cells by capsaicin. Chin Med J 90: 1230-1233, 2010 (In Chinese).

35. Mori A, Lehmann S, O'Kelly J, Kumagai T, Desmond JC, Pervan M, McBride WH, Kizaki M and Koeffler HP Capsaicin, a component of red peppers, inhibits the growth of androgen-independent, p53 mutant prostate cancer cells. Cancer Res 66: 3222-3229, 2006.

36. Bunch RT and Eastman A: 7-Hydroxystaurosporine (UCN-01) causes redistribution of proliferating cell nuclear antigen and abrogates cisplatin-induced S-phase arrest in Chinese hamster ovary cells. Cell Growth Differ 8: 779-788, 1997.

37. Koundrioukoff S, Jónsson ZO, Hasan S, de Jong RN, van der Vliet PC, Hottiger MO and Hübscher U: A direct interaction between proliferating cell nuclear antigen (PCNA) and Cdk2 targets PCNA-interacting proteins for phosphorylation. J Biol Chem 275: 22882-22887, 2000.

38. Moldovan GL, Pfander B and Jentsch S: PCNA controls establishment of sister chromatid cohesion during $\mathrm{S}$ phase. Mol Cell 23: 723-732, 2006

39. Yoshikawa H, Ikeuchi $\mathrm{T}$ and Kai Y: Immunohistochemical study of the proliferating ability and malignant potential of transitional cell carcinoma in the human urinary bladder. Int J Urol 3 (Suppl 1): S32-S34, 1996.

40. Inagaki T, Ebisuno S, Uekado Y, Hirano A, Hiroi A, Shinka T and Ohkawa T: PCNA and p53 in urinary bladder cancer: Correlation with histological findings and prognosis. Int $\mathrm{J}$ Urol 4: 172-177, 1997.

41. Tong Q, Zeng F, Lin C, Zhao J and Lu G: Growth inhibiting effects of antisense eukaryotic expression vector of proliferating cell nuclear antigen gene on human bladder cancer cells. Chin Med J (Engl) 116: 1203-1206, 2003.

42. Zhu Z, Xing S, Lin C, Zhang X, Fu M, Liang X, Zeng F, $\mathrm{Lu} \mathrm{G}$ and $\mathrm{Wu} \mathrm{M}$ : Bladder cancer therapy using combined proliferating cell nuclear antigen antisense oligonucleotides and recombinant adenovirus p53. Chin Med J (Engl) 116 1860-1863, 2003.

43. Bodó E, Bíró T, Telek A, Czifra G, Griger Z, Tóth BI, Mescalchin A, Ito T, Bettermann A, Kovács L and Paus R: A hot new twist to hair biology: Involvement of vanilloid receptor-1 (VR1/TRPV1) signaling in human hair growth control. Am J Pathol 166: 985-998, 2005.

44. de Jong PR, Takahashi N, Harris AR, Lee J, Bertin S, Jeffries J, Jung M, Duong J, Triano AI, Lee J, et al: Ion channel TRPV1-dependent activation of PTP1B suppresses EGFR-associated intestinal tumorigenesis. J Clin Invest 124: 3793-3806, 2014. 